\title{
The Agriculture and Forestry Lands Level of Changes in Planning Process as a Sustainable Development Measure of Municipalities Located within National Parks Impact Areas
}

\author{
Konrad Podawca ${ }^{1 *}$, Agata Pawłat-Zawrzykraj ${ }^{1}$ \\ 1 Faculty of Civil and Environmental Engineering, Warsaw University of Life Sciences - SGGW, ul. \\ Nowoursynowska 159, 02-776 Warsaw, Poland \\ * Corresponding author's e-mail: konrad_podawca@sggw.pl
}

\begin{abstract}
The change in the use of agricultural and forestry land for other purposes is the natural process of rural areas urbanisation. In the municipalities located nearby large cities, this transformation is inevitable and indeed desirable, while in the municipalities located close to the protected areas, it is a symptom of negative processes, especially from an environmental perspective. Moreover, such transformations have many other economic and social consequences which are worth paying special attention to. This paper is an attempt to determine the level of negative changes introduced in the planning documents in municipalities located within the borders of National Parks. The indicator analysis based on the statistical data of local date bank [BDL] in the fields of local government and spatial planning was conducted. The analysis enabled to present the differences between municipalities with very high natural values in terms of decreasing the area of agricultural and forestry landscape. Furthermore, to elaborate the typology of the studied administrative units the obtained results were verified by comparing them with recommended percentage share of forest and agricultural lands which potentially guarantee good conditions for the development of tourism in rural municipalities.
\end{abstract}

Keywords: changing the status of agricultural and forest land, municipality, sustainable development, spatial planning

\section{INTRODUCTION}

By 2016, the interest in purchasing the agricultural and forestry lands for construction purposes increased. It was driven both by economical and sociological aspects. An important factor was a lower price of the agricultural and forestry lands, in comparison to the construction plots. This kind of purchase was often treated as an investment or a good place to live, due to the pollution-free environment and distance from the issues of large cities (Kostańska, 2018). The common practice of purchasing agricultural lands was restricted by the Act of 14 April 2016 on suspension of the sale of real property from the Agricultural Property Stock (Journal of Laws of 2018 Item. 869). The Act introduced a number of regulations, in order to protect the agricultural lands from speculative redemption and to ensure that such lands will be used for the agricultural purpose. Under these circumstances, the role of planning procedures increased, as well as the enactment of local spatial development plans in which the agricultural and forest lands might have been changed for other purposes. These documents reduced the restrictions imposed on the buyer of the agricultural lands (Suchoń, 2016). Additionally, in March 2019, the Government adopted the draft Amending the Act on Shaping of the Agricultural System, which assumes alleviating the limitations in trading the agricultural real estates. However, this may lead to repeated increase purchases of agricultural lands which would be subsequently converted into non-agricultural purposes.

Sustainable development should be a priority taken into account on every stage of the 
planning process in a municipality (Chmielews$\mathrm{ki}, 2001)$. The processes related to changing the status of agricultural and forestry lands are important factors that will affect the spatial structure of the area, and consequently, such impact factors as: biodiversity, landscape, pollution and touristic values. According to many authors and experts, there is a correlation between changing the agricultural and forestry land use for other purposes, chaos in rural space and fragmentation of agricultural landscape (Kołodziejczak and Kaczmarek, 2018). Therefore, the issues of proper spatial management of rural areas, remain valid and relevant today This kind of discussions have taken place for several years not only among the experts on spatial planning, local authorities and communities but also at the higher political level. For instance, in June 2016, in the Ministry of Agriculture and Rural Development a seminar was held on the need for amending spatial planning of rural areas. Additionally, in March 2019, the Supreme Chamber of Control organized a conference on social, economic and environmental dimension of Polish space management crisis (Wilkin, 2018; Kołodziejczak and al., 2018). The conclusions drawn from the discussions mentioned above, mainly pointed out the lack of effectiveness of planning structures and challenges that the public institutions face in terms of effective rural area development (Kwartnik-Pruc and Parzych and Bydłosz, 2011; Krzysztofik 2016). In theory, the objective of the current rural development policy is to preserve and develop the natural, cultural and landscape values of rural areas (Heffner and Klemens, 2016). Studies were conducted to evaluate the implementation of such priorities, mainly in the aspect of planning process in the municipalities, whose common feature is that they are located within the large-scale areas of nature protection.

\section{GOAL AND SCOPE OF THE STUDY}

The cognitive goal of the study is to show the differentiation from the municipalities located within impact area of National Parks, in terms of the intensity of changes in the use of agricultural and forestry land for other purposes in the spatial planning process. The research covered 113 municipalities, including 75 rural, 27 urbanrural and 11 urban ones. The municipality of
Nowinka was eliminated from the research due to incorrect data in BDL.

The authors put forward the hypothesis that intensification of the planning decisions related to the change in the use of agricultural and forest lands for non-agricultural and non-forest purposes in the municipalities located within the impact area of National Parks is low. This assumption is in line with protecting agricultural and forest landscape on the examined areas, coming from high environmental values.

The selected municipalities were already the subject of studies conducted both by the authors (Pawłat-Zawrzykraj and Podawca, 2011; Podawca, 2014; Podawca, 2015; Podawca and Karsznia, 2017; Podawca and Pawłat-Zawrzykraj, 2017a; Podawca and Pawłat-Zawrzykraj, 2017b; Podawca and Pawłat-Zawrzykraj, 2018) as well as other researchers (Giordano, 2006; Liszewski 2009; Zawilińska and Mika, 2013). The studies concerned all national parks or particular municipalities. mainly natural values, various aspects of tourism development, technical infrastructure, quality of life and broadly understood environment protection issues. The impact of planning decisions on the transformation of the spatial structure, including those concerning the changes of agricultural and forestry land for other purposes, was analyzed rather in the local dimension, usually for the communities located nearby large cities (Tanaś, 2014; Grochowska, 2016; Sawicka and Fogel, 2016; Kołodziejczak and Kacprzak, 2016; Markuszewska and Marchewka, 2016; Kołodziejczak, 2017; Podawca and Mrozik, 2019). Therefore, taking up this issue for the specific aggregation of municipalities associated with National Parks appears reasonable.

Showing the quantitative aspects of changes in land-use for other than agricultural and forest functions and primarily the standardization of the obtained data owing to the proposed indicators will help to create the typology of the administrative units. Such typology, is also the empirical goal of the research that seeks to extract the municipalities, where the process of land use changes concerning the agricultural and forestry areas may bring about adverse changes of spatial structure that affect tourist development, nature conservation, as well as sustainable and ecological development in general. 


\section{METHODS}

The method of the study involves comparative analysis using specific indicators that are unable to describe intensity of the phenomena in absolute or relative values (Zielińska, 2013). This type of analysis is a commonly used tool for the interpretation of the spatial information, including the aspects of eco-development (Borys, 1999). The evaluation of municipalities according to the sustainable development indicators is not a new issue. The literature concerns both the methodology as well as research aspect (Rosner, 1999; Giordano, 2006; Śleszyński, 2013). However, the proposed set of indicators and the idea of using them to expend the evaluation the municipalities spatially and functionally linked to National Parks is the authors' contribution.

The methods adopted in the paper can be divided into two groups:

- the analysis of statistical data collected in local data bank in the fields of local government and spatial planning,

- data processing of negative variables which means that low values indicate better sustainable development of the analyzed municipalities.

It was recognized that the quantity of landuse changes for non-agricultural and non-forestry purposes in the planning process would be described by total area of agricultural land, changed for non-agricultural $\left(\mathrm{P}_{\text {cha }}\right)$ and by total area of forest lands for which the use in the plans was changed for non-forest $\left(\mathrm{P}_{\text {chf }}\right)$.

The selected features, depending on their character, were related to the total area of agricultural $\left(\mathrm{P}_{\mathrm{a}}\right)$ or forest lands $\left(\mathrm{P}_{\mathrm{f}}\right)$ and the total area of the municipality covered with local spatial development plans. This kind of approach should eliminate the size issue of a municipality and enable comparison between the administration units.

The measurement of changes in the agricultural and forestry lands use for the other purposes includes:

- the absolute degree of changes of agricultural (forestry) lands for non-agricultural (non-forest) purposes described as:

$$
W_{c h a(c h f)}=\left(\frac{P_{c h a(c h f)}}{P_{a(f)}}\right) x 100
$$

where: $W_{\text {cha(chf) }}$-indicator of changes in the area of agricultural (forest) lands in 2014 [\%];
$P_{\text {cha(chf) }}$ - the total area of agricultural (forest) lands covered with changes for non-agricultural (non-forest) purposes by local spatial development plans [ha];

$P_{a(f)}$ - area of agricultural (forest) lands in the municipality as of 2014 [ha];

- the relative changes degree of an area of agricultural (forestry) lands described as:

$$
W_{p a(p f)}=\left(\frac{P_{c h a(c h f)}}{P_{l s d p}}\right) x 100
$$

where: $W_{p a(p f)}-$ the indicator of spatial planning changes in destination of agricultural (forest) lands in 2014 [\%];

$P_{\text {cha(chf) }}$ - the total area of agricultural (forest) lands covered with changes for non-agricultural (non-forest) purposes by local spatial development plans [ha];

$P_{\text {Isdp }}$ - the total area of a municipality covered with local spatial development plans [ha].

The limit values that characterize agricultural and forest lands were used for the assessment of the sustainability and conditions for tourism development of the municipalities in terms of the decreasing area of rural and forest landscape.

According to the fundamental assessment methods of the areas with valuable natural assets for sustainable tourism development (mainly agri-tourism) it was recognized that:

- the share of agricultural lands in total municipality area should be in the range of $25-30 \%$,

- forestry shares totalized municipality area should be in the range of 30-60\% (Drzewiecki, 1992; Drzewiecki, 2005).

- Therefore, it was decided to present the relation between reduction of agricultural and forest lands and sustainable development of the municipalities by using the following coefficients:

- Spatial coefficient of agricultural landscape $\mathrm{W}_{\mathrm{la}}[\%]$

$$
W_{l a}=\left[\left(\frac{P_{a}-P_{c h a}}{P_{m}}\right)-0.25\right] x 100
$$

where: 0.25 - minimal agricultural land shares in a rural municipality with high natural values; 
$P_{a}-$ agricultural land shares in the municipality as at 2014 [ha];

$P_{c h a}-$ the total agricultural land areas covered with changes for non-agricultural purposes by local spatial development plans [ha];

$P_{m}-$ municipality total area [ha].

- Forestry landscape Spatial coefficient $\mathrm{W}_{\mathrm{If}}$

$$
W_{l f}=\left[\left(\frac{P_{f}-P_{c h f}}{P_{m}}\right)-0.30\right] x 100
$$

where: $0.30-$ minimal forestry land shares in rural municipality with high natural values; $P_{f}$ - forestry lands area in a municipality as at 2014 [ha];

$P_{c h f}$-forestry land total area covered with changes for non-forest purposes by local spatial development plans [ha];

$P_{m}$-municipality total area [ha].

The variables presented above are universal, measurable, have good data availability and will enable to compare indicators and present an objective interpretation of the obtained results. They are correct from theoretical perspective and are applicable not only for planning research procedure but also in evaluation of other administration units.

Particular spatial determination of parameters and data analysis was done using the data from the local data bank (BDL) and ArcGis software.

\section{RESULTS AND DISCUSSION}

According to the presented method, the relative degree changes of the area in forestry lands is supposed to show to what extent the local spatial development plans are designed to amend the role of forests. In the analyzed set of municipalities, the indicator value levels are extremely low, which proves the good planning protection of the forests in the examined cases. In 71 units $(62.8 \%)$ there was no change in forest function. In 32 municipalities (28.3\%) less than 2 ha of forest out of the 100 ha of total area covered with local spatial development plans were designated for the non-forest purposes. Between 2 and 5 ha were designated in 6 municipalities $(5.3 \%)$ and between 5 and 10 ha in 3 cases. Highly negative values of the indicator were observed in the Białowieża municipality where $28.5 \%$ of the total area covered with the plans concerning forestry land transformations. However, it should be mentioned that the total area covered with spatial development plans in this administrative unit is small. A similar situation is observed for the absolute degree of forestry land changes. The calculated values are low. This is a very good situation regarding sustainable development and tourist attractiveness. In 71 units $(62.8 \%)$ there were no changes concerning the forestry land. In 35 municipalities $(31 \%)$ the indicator was below 1 , which means that the planning documents were less than 1 ha for every 100 ha of forest that was changed for the non-forestry purposes. 5 municipalities (4.4\%) changed up to 2 ha on every 100 ha for forest lands and in one case the area was slightly above 2 ha $(0.9 \%)$. A special case is the Łapy municipality where 8 out of 100 ha was changed for non-forestry purposes (Table 1).

The planning decisions, from the agricultural lands protection perspective, look different. Taking into consideration both relative and absolute degree of the analyzed land-use changes only in 38 municipalities (33.6\%) there were no provisions in local spatial development plans on the use of agricultural land. Regarding the changes in the absolute degrees of agricultural land, in large number of the units up to $10 \%$ of the total area covered with local spatial development plans constituted changes in use of agricultural land. From 10 to 20 ha of agricultural land per 100 ha covered with plans was changed for nonagricultural purposes in 11 municipalities (9.7\%), from 20 ha to 30 ha in 8 cases $(7.1 \%)$, more than 30 ha in $16(14.2 \%)$ municipalities, whereas in 11 cases more than 80 ha, which means that the local spatial developments plans were mainly aimed at changing the use of agricultural lands. Taking into consideration the absolute degree of changes of agricultural land for non-agricultural purposes, up to 2 ha per 100 ha of agricultural land was changed in 43 municipalities (38.1\%), the area between 2 ha and 5 ha in 10 administrative units (8.85\%), between 5 ha and 10 ha in 8 units, whereas the range between 10 ha 20 ha - in the case of 10 municipalities.

The agricultural land changes for other purposes in relation to the total agricultural areas of more than $20 \%$ were investigated in 4 municipalities (3.5\%). Highly negative results were obtained for Zakopane and Karpacz. However, these are the cases of urban municipalities where regulations related to agricultural lands are less 
Table 1. Input data and obtained indicators concerning changes of agricultural and forestry land for other purposes in the analyzed municipalities (own elaboration)

\begin{tabular}{|c|c|c|c|c|c|c|c|c|c|c|c|c|c|c|}
\hline \multirow{2}{*}{ No. } & \multirow{2}{*}{ Municipality } & \multirow{2}{*}{ PN } & \multirow{2}{*}{$P_{m}\left[\mathrm{~km}^{2}\right]$} & \multirow{2}{*}{$\begin{array}{c}\mathrm{P}_{\mathrm{a}} \\
\text { [ha] }\end{array}$} & \multirow{2}{*}{$\begin{array}{c}\mathrm{P}_{\mathrm{f}} \\
{[\text { ha] }}\end{array}$} & \multirow{2}{*}{$\begin{array}{l}P_{\text {cha }} \\
\text { [ha] }\end{array}$} & \multirow{2}{*}{$\begin{array}{l}P_{\text {chf }} \\
\text { [ha] }\end{array}$} & \multirow{2}{*}{$\begin{array}{l}\mathrm{P}_{\text {Issp }} \\
\text { [ha] }\end{array}$} & \multicolumn{6}{|c|}{ Indicators of land-use changes [\%] } \\
\hline & & & & & & & & & $W_{\text {cha }}$ & $W_{\mathrm{chf}}$ & $\mathrm{W}_{\mathrm{pa}}$ & $W_{p f}$ & $\mathrm{~W}_{\mathrm{la}}$ & $\mathrm{W}_{\mathrm{lf}}$ \\
\hline 1 & Górzyca & & 145.42 & 9641 & 3447 & 380 & 0 & 1810 & 3.941 & 0 & 20.994 & 0 & 41.30 & -6.30 \\
\hline 2 & Kostrzyn n.Odrą* & & 46.14 & 1055 & 1838 & 0 & 35 & 1040 & 0 & 1.904 & 0 & 3.365 & -2.13 & 9.84 \\
\hline 3 & Witnica & & 278.68 & 12245 & 12548 & 44 & 1 & 214 & 0.359 & 0.008 & 20.561 & 0.467 & 18.94 & 15.03 \\
\hline 4 & Słońsk & & 158.64 & 10502 & 3633 & 0 & 0 & 20 & 0 & 0 & 0 & 0 & 41.20 & -7.10 \\
\hline 5 & Lipnica Wielka & \multirow{2}{*}{$=$} & 67.36 & 3218 & 3030 & 340 & 0 & 6747 & 10.566 & 0 & 5.039 & 0 & 22.77 & 14.98 \\
\hline 6 & Zawoja & & 128.78 & 4020 & 8405 & 254 & 36 & 12866 & 6.318 & 0.428 & 1.974 & 0.280 & 6.22 & 35.27 \\
\hline 7 & Narewka & \multirow{2}{*}{$\equiv$} & 338.98 & 8506 & 23121 & 222 & 32 & 724 & 2.610 & 0.138 & 30.663 & 4.420 & 0.09 & 38.21 \\
\hline 8 & Białowieża & & 203.14 & 1514 & 18041 & 0 & 6 & 21 & 0 & 0.033 & 0 & 28.571 & -17.55 & 58.81 \\
\hline 9 & Wizna & \multirow{14}{*}{$\geq$} & 133.38 & 11031 & 1379 & 12 & 0 & 37 & 0.109 & 0 & 32.432 & 0 & 57.70 & -19.66 \\
\hline 10 & Nowy Dwór & & 121.14 & 9718 & 1730 & 318 & 0 & 356 & 3.272 & 0 & 89.326 & 0 & 55.22 & -15.72 \\
\hline 11 & Bargłów Kościelny & & 187.81 & 14161 & 2552 & 63 & 0 & 318 & 0.445 & 0 & 19.811 & 0 & 50.40 & -16.41 \\
\hline 12 & Jedwabne & & 159.21 & 12380 & 2831 & 15 & 1 & 22 & 0.121 & 0.035 & 68.182 & 4.545 & 52.76 & -12.22 \\
\hline 13 & Grajewo & & 308.23 & 18753 & 9845 & 362 & 10 & 2951 & 1.930 & 0.102 & 12.267 & 0.339 & 35.84 & 1.94 \\
\hline 14 & Jaświły & & 175.49 & 15477 & 1268 & 55 & 2 & 17549 & 0.355 & 0.158 & 0.313 & 0.011 & 63.19 & -22.77 \\
\hline 15 & Rajgród & & 207.26 & 12459 & 5873 & 186 & 8 & 1893 & 1.493 & 0.136 & 9.826 & 0.423 & 35.11 & -1.66 \\
\hline 16 & Lipsk & & 184.21 & 12068 & 4199 & 31 & 0 & 31 & 0.257 & 0 & 100 & 0 & 40.51 & -7.21 \\
\hline 17 & $\begin{array}{l}\text { Dabbrowa } \\
\text { Białostocka }\end{array}$ & & 263.84 & 20404 & 4101 & 4 & 0 & 2265 & 0.020 & 0 & 0.177 & 0 & 52.33 & -14.46 \\
\hline 18 & Suchowola & & 256.7 & 20059 & 2720 & 0 & 0 & 0 & 0 & 0 & $0^{*}$ & $0^{*}$ & 53.45 & -19.36 \\
\hline 19 & Radziłów & & 199.54 & 15082 & 1884 & 46 & 0 & 2083 & 0.305 & 0 & 2.208 & 0 & 50.58 & -20.56 \\
\hline 20 & Sztabin & & 363.11 & 18380 & 15424 & 85 & 9 & 441 & 0.462 & 0.058 & 19.274 & 2.041 & 25.62 & 12.48 \\
\hline 21 & Trzcianne & & 331.87 & 14843 & 6854 & 1 & 0 & 1 & 0.007 & 0 & 100 & 0 & 19.73 & -9.35 \\
\hline 22 & Goniądz & & 376.58 & 13969 & 11986 & 137 & 0 & 37668 & 0.981 & 0 & 0.364 & 0 & 12.09 & 1.83 \\
\hline 23 & Czarna & \multirow{3}{*}{$>$} & 184.77 & 4511 & 11542 & 18 & 0 & 112 & 0.399 & 0 & 16.071 & 0 & -0.59 & 32.47 \\
\hline 24 & Cisna & & 287.26 & 1586 & 25519 & 0 & 0 & 789 & 0 & 0 & 0 & 0 & -19.48 & 58.84 \\
\hline 25 & Lutowiska & & 475.63 & 4565 & 39327 & 80 & 0 & 722 & 1.752 & 0 & 11.080 & 0 & -15.40 & 52.68 \\
\hline 26 & Chojnice & 5 & 458.21 & 22232 & 17799 & 1 & 0 & 3430 & 0.004 & 0 & 0.029 & 0 & 23.52 & 8.84 \\
\hline 27 & Bierzwnik & \multirow{6}{*}{$\bar{\Sigma}$} & 239.06 & 8630 & 12916 & 29 & 0 & 67 & 0.336 & 0 & 43.284 & 0 & 11.10 & 24.03 \\
\hline 28 & Krzyż Wielkopolski & & 174.28 & 5987 & 10190 & 34 & 3 & 1497 & 0.568 & 0.029 & 2.271 & 0.200 & 9.35 & 28.47 \\
\hline 29 & Tuczno & & 249.5 & 9841 & 12474 & 0 & 0 & 326 & 0 & 0 & 0 & 0 & 14.44 & 20.00 \\
\hline 30 & Drawno & & 320.19 & 7180 & 22350 & 0 & 0 & 235 & 0 & 0 & 0 & 0 & -2.63 & 39.65 \\
\hline 31 & Człopa & & 349.05 & 7146 & 25539 & 45 & 0 & 45 & 0.630 & 0 & 100 & 0 & -4.53 & 43.17 \\
\hline 32 & Dobiegniew & & 351.27 & 9278 & 21684 & 10 & 5 & 843 & 0.108 & 0.023 & 1.186 & 0.593 & 1.41 & 31.73 \\
\hline 33 & Nowy Targ & \multirow{5}{*}{$\overline{\bar{j}}$} & 207.68 & 11838 & 7578 & 0 & 0 & 3355 & 0 & 0 & 0 & 0 & 32.00 & 6.49 \\
\hline 34 & Ochotnica Dolna & & 141.2 & 5505 & 8177 & 0 & 0 & 14100 & 0 & 0 & 0 & 0 & 13.99 & 27.91 \\
\hline 35 & Mszana Dolna & & 170.02 & 8686 & 7218 & 0 & 1 & 17002 & 0 & 0.014 & 0 & 0.006 & 26.09 & 12.45 \\
\hline 36 & Kamienica & & 95.18 & 3116 & 5924 & 448 & 2 & 4863 & 14.377 & 0.034 & 9.212 & 0.041 & 7.74 & 32.24 \\
\hline 37 & Niedźwiedź & & 74.22 & 2928 & 4054 & 50 & 0 & 7444 & 1.708 & 0 & 0.672 & 0 & 14.45 & 24.62 \\
\hline 38 & Lewin Kłodzki & \multirow{4}{*}{$\underline{x}$} & 52.14 & 2413 & 2463 & 0 & 0 & 157 & 0 & 0 & 0 & 0 & 21.28 & 17.24 \\
\hline 39 & Kudowa Zdrój* & & 33.9 & 1315 & 1621 & 8 & 0 & 3387 & 0.608 & 0 & 0.236 & 0 & 13.79 & 17.82 \\
\hline 40 & Szczytna & & 132.37 & 4075 & 8548 & 128 & 0 & 13202 & 3.141 & 0 & 0.970 & 0 & 5.78 & 34.58 \\
\hline 41 & Radków & & 139.95 & 7998 & 5094 & 518 & 17 & 13962 & 6.477 & 0.334 & 3.710 & 0.122 & 32.15 & 6.40 \\
\hline
\end{tabular}


Table 1 cont.

\begin{tabular}{|c|c|c|c|c|c|c|c|c|c|c|c|c|c|c|}
\hline \multirow{2}{*}{ No. } & \multirow{2}{*}{ Municipality } & \multirow{2}{*}{ PN } & \multirow{2}{*}{$P_{m}\left[\mathrm{~km}^{2}\right]$} & \multirow{2}{*}{$\begin{array}{c}\mathrm{P}_{\mathrm{a}} \\
{[\mathrm{ha}]}\end{array}$} & \multirow{2}{*}{$\begin{array}{c}\mathrm{P}_{\mathrm{f}} \\
{[\mathrm{ha}]}\end{array}$} & \multirow{2}{*}{$\begin{array}{l}\mathrm{P}_{\text {cha }} \\
\text { [ha] }\end{array}$} & \multirow{2}{*}{$\begin{array}{l}P_{\text {chf }} \\
\text { [ha] }\end{array}$} & \multirow{2}{*}{$\begin{array}{l}\mathrm{P}_{\text {Isdo }} \\
\text { [ha] }\end{array}$} & \multicolumn{6}{|c|}{ Indicators of land-use changes [\%] } \\
\hline & & & & & & & & & $W_{\text {cha }}$ & $\mathrm{W}_{\mathrm{chf}}$ & $\mathrm{W}_{\mathrm{pa}}$ & $W_{p f}$ & $\mathrm{~W}_{\text {la }}$ & $\mathrm{W}_{\text {Iff }}$ \\
\hline 42 & $\begin{array}{l}\text { Tomaszów } \\
\text { Mazowiecki }\end{array}$ & \multirow{9}{*}{$\lambda$} & 149.81 & 6669 & 6732 & 0 & 105 & 1224 & 0 & 1.560 & 0 & 8.578 & 19.51 & 14.93 \\
\hline 43 & Łomianki & & 38.83 & 1721 & 595 & 184 & 12 & 709 & 10.691 & 2.017 & 25.952 & 1.693 & 19.32 & -14.68 \\
\hline 44 & Stare Babice & & 63.42 & 4443 & 1227 & 1731 & 5 & 6342 & 38.960 & 0.407 & 27.294 & 0.079 & 45.06 & -10.65 \\
\hline 45 & Kampinos & & 84.6 & 6110 & 1748 & 762 & 0 & 762 & 12.471 & 0 & 100 & 0 & 47.22 & -9.34 \\
\hline 46 & Brochów & & 119.81 & 6335 & 4019 & 67 & 0 & 67 & 1.058 & 0 & 100 & 0 & 27.88 & 3.54 \\
\hline 47 & Izabelin & & 65.01 & 578 & 5036 & 95 & 55 & 880 & 16.436 & 1.092 & 10.795 & 6.250 & -16.11 & 47.47 \\
\hline 48 & Czosnów & & 128.45 & 7123 & 3593 & 1240 & 11 & 12845 & 17.408 & 0.306 & 9.654 & 0.086 & 30.45 & -2.03 \\
\hline 49 & Leszno & & 125.08 & 6424 & 5214 & 352 & 15 & 3160 & 5.479 & 0.288 & 11.139 & 0.475 & 26.36 & 11.69 \\
\hline 50 & Leoncin & & 157.98 & 6121 & 8063 & 769 & 3 & 2755 & 12.563 & 0.037 & 27.913 & 0.109 & 13.75 & 21.04 \\
\hline 51 & Kowary* & \multirow{6}{*}{$\bar{x}$} & 37.39 & 837 & 2438 & 45 & 24 & 3738 & 5.376 & 0.984 & 1.204 & 0.642 & -2.61 & 35.20 \\
\hline 52 & Piechowice* & & 43.22 & 963 & 2748 & 0 & 0 & 2100 & 0 & 0 & 0 & 0 & -2.72 & 33.58 \\
\hline 53 & Podgórzyn & & 82.51 & 3079 & 4165 & 0 & 0 & 4320 & 0 & 0 & 0 & 0 & 12.32 & 20.48 \\
\hline 54 & Szklarska Poręba* & & 75.44 & 433 & 6191 & 0 & 7 & 995 & 0 & 0.113 & 0 & 0.704 & -19.26 & 52.07 \\
\hline 55 & Jelenia Góra* & & $\mid 109.22$ & 4331 & 3628 & 0 & 0 & 9717 & 0 & 0 & 0 & 0 & 14.65 & 3.22 \\
\hline 56 & Karpacz* & & 37.99 & 392 & 2487 & 392 & 13 & 2016 & 100 & 0.523 & 19.444 & 0.645 & -14.68 & 35.46 \\
\hline 57 & Osiek Jasielski & \multirow{6}{*}{$\overline{\bar{x}}$} & 60.4 & 3746 & 1876 & 55 & 0 & 966 & 1.468 & 0 & 5.694 & 0 & 37.02 & 1.06 \\
\hline 58 & Sękowa & & 194.8 & 5252 & 13611 & 0 & 0 & 19480 & 0 & 0 & 0 & 0 & 1.96 & 39.87 \\
\hline 59 & Lipinki & & 66.46 & 4265 & 2076 & 35 & 1 & 6616 & 0.821 & 0.048 & 0.529 & 0.015 & 39.17 & 1.24 \\
\hline 60 & Nowy Żmigród & & 103.59 & 6731 & 3031 & 0 & 0 & 10290 & 0 & 0 & 0 & 0 & 39.98 & -0.74 \\
\hline 61 & Dębowiec & & 86.47 & 4983 & 2975 & 10 & 0 & 7734 & 0.201 & 0 & 0.129 & 0 & 32.63 & 4.40 \\
\hline 62 & Krempna & & 203.86 & 4042 & 15361 & 0 & 0 & 2 & 0 & 0 & 0 & 0 & -5.17 & 45.35 \\
\hline 63 & Tykocin & \multirow{7}{*}{$\overline{\bar{x}}$} & 207.37 & 13464 & 5509 & 68 & 0 & 68 & 0.505 & 0 & 100 & 0 & 39.93 & -3.43 \\
\hline 64 & Kobylin-Borzymy & & $\mid 119.42$ & 9068 & 2176 & 20 & 0 & 577 & 0.221 & 0 & 3.466 & 0 & 50.93 & -11.78 \\
\hline 65 & Suraż & & 76.61 & 6102 & 1043 & 0 & 0 & 61 & 0 & 0 & 0 & 0 & 54.65 & -16.39 \\
\hline 66 & Sokoły & & 155.6 & 11129 & 3040 & 94 & 4 & 106 & 0.845 & 0.132 & 88.679 & 3.774 & 46.52 & -10.46 \\
\hline 67 & Turośń Kościelna & & 139.9 & 8995 & 3270 & 0 & 0 & 257 & 0 & 0 & 0 & 0 & 39.30 & -6.63 \\
\hline 68 & Choroszcz & & \begin{tabular}{|l|}
163.79 \\
\end{tabular} & 10138 & 2715 & 0 & 0 & 16370 & 0 & 0 & 0 & 0 & 36.90 & -13.42 \\
\hline 69 & Łapy & & 127.65 & 8121 & 1737 & 60 & 137 & 12757 & 0.739 & 7.887 & 0.470 & 1.074 & 38.62 & -16.39 \\
\hline 70 & Wielka Wieś & \multirow{4}{*}{$\vec{x}$} & 48.27 & 3947 & 363 & 0 & 0 & 4690 & 0 & 0 & 0 & 0 & 56.77 & -22.48 \\
\hline 71 & $\begin{array}{l}\text { Jerzmanowice- } \\
\text { Przeginia }\end{array}$ & & 68.14 & 5930 & 610 & 1 & 0 & 6839 & 0.017 & 0 & 0.015 & 0 & 62.03 & -21.05 \\
\hline 72 & Sułoszowa & & 53.38 & 4837 & 371 & 128 & 0 & 5146 & 2.646 & 0 & 2.487 & 0 & 65.61 & -23.05 \\
\hline 73 & Skała & & 74.83 & 5577 & 1570 & 0 & 0 & 3529 & 0 & 0 & 0 & 0 & 49.53 & -9.02 \\
\hline 74 & Łapsze Niżne & \multirow{4}{*}{$\vec{x}$} & 125.79 & 6852 & 4681 & 75 & 0 & 12500 & 1.095 & 0 & 0.600 & 0 & 29.47 & 7.21 \\
\hline 75 & Szczawnica & & 87.9 & 2132 & 6030 & 0 & 0 & 1040 & 0 & 0 & 0 & 0 & -0.75 & 38.60 \\
\hline 76 & $\begin{array}{l}\text { Krościenko nad } \\
\text { Dunajcem }\end{array}$ & & 57.12 & 2561 & 2847 & 91 & 0 & 1839 & 3.553 & 0 & 4.948 & 0 & 19.84 & 19.84 \\
\hline 77 & Czorsztyn & & 62.16 & 2347 & 2836 & 171 & 0 & 5018 & 7.286 & 0 & 3.408 & 0 & 12.76 & 15.62 \\
\hline 78 & Ludwin & \multirow{6}{*}{$\sum_{x}$} & 122.17 & 8508 & 1669 & 315 & 4 & 12122 & 3.702 & 0.240 & 2.599 & 0.033 & 44.64 & -16.34 \\
\hline 79 & Stary Brus & & 131.67 & 6167 & 6111 & 0 & 0 & 0 & 0 & 0 & $0^{*}$ & $0^{*}$ & 21.84 & 16.41 \\
\hline 80 & Hańsk & & \begin{tabular}{|l|}
176.27 \\
\end{tabular} & 8742 & 6936 & 7 & 0 & 302 & 0.080 & 0 & 2.318 & 0 & 24.59 & 9.35 \\
\hline 81 & Wierzbica & & 145.79 & 11992 & 1326 & 0 & 0 & 14636 & 0 & 0 & 0 & 0 & 57.26 & -20.90 \\
\hline 82 & Sosnowica & & $\mid 171.62$ & 7341 & 7711 & 91 & 0 & 91 & 1.240 & 0 & 100 & 0 & 17.77 & 14.93 \\
\hline 83 & Urszulin & & 172.14 & 9319 & 4622 & 23 & 0 & 150 & 0.247 & 0 & 15.333 & 0 & 29.14 & -3.15 \\
\hline 84 & Zamość & \multirow{4}{*}{$\bar{x}$} & $\mid 196.11$ & 16496 & 1754 & 522 & 0 & 1888 & 3.164 & 0 & 27.648 & 0 & 59.12 & -21.06 \\
\hline 85 & Józefów & & 126.46 & 4903 & 7307 & 0 & 0 & 12452 & 0 & 0 & 0 & 0 & 13.77 & 27.78 \\
\hline 86 & Adamów & & \begin{tabular}{|l|}
110.66 \\
\end{tabular} & 6117 & 4710 & 923 & 0 & 11055 & 15.089 & 0 & 8.349 & 0 & 30.28 & 12.56 \\
\hline 87 & Zwierzyniec & & 153.55 & 3727 & 11000 & 11 & 3 & 1280 & 0.295 & 0.027 & 0.859 & 0.234 & -0.73 & 41.64 \\
\hline
\end{tabular}


Table 1 cont.

\begin{tabular}{|c|c|c|c|c|c|c|c|c|c|c|c|c|c|c|}
\hline \multirow{2}{*}{ No. } & \multirow{2}{*}{ Municipality } & \multirow{2}{*}{ PN } & \multirow{2}{*}{$P_{m}\left[k^{2}\right]$} & \multirow{2}{*}{$\begin{array}{c}\mathrm{P}_{\mathrm{a}} \\
\text { [ha] }\end{array}$} & \multirow{2}{*}{$\begin{array}{c}\mathrm{P}_{\mathrm{f}} \\
\text { [ha] }\end{array}$} & \multirow{2}{*}{$\begin{array}{l}P_{\text {cha }} \\
\text { [ha] }\end{array}$} & \multirow{2}{*}{$\begin{array}{l}P_{\text {chf }} \\
\text { [ha] }\end{array}$} & \multirow{2}{*}{$\begin{array}{l}P_{\text {Isdp }} \\
\text { [ha] }\end{array}$} & \multicolumn{6}{|c|}{ Indicators of land-use changes [\%] } \\
\hline & & & & & & & & & $\mathrm{W}_{\text {cha }}$ & $W_{\text {chf }}$ & $W_{p a}$ & $W_{p f}$ & $\mathrm{~W}_{\mathrm{la}}$ & $\mathrm{W}_{\mathrm{lf}}$ \\
\hline 88 & Ustka & \multirow{5}{*}{$\bar{\equiv}$} & 217.46 & 12183 & 6732 & 1102 & 74 & 4745 & 9.045 & 1.099 & 23.224 & 1.560 & 31.02 & 0.96 \\
\hline 89 & Łeba* & & 14.81 & 248 & 701 & 2 & 8 & 193 & 0.806 & 1.141 & 1.036 & 4.145 & -8.25 & 17.33 \\
\hline 90 & Główczyce & & 321.97 & 18762 & 9850 & 92 & 0 & 4004 & 0.490 & 0 & 2.298 & 0 & 33.27 & 0.59 \\
\hline 91 & Wicko & & 215.29 & 10681 & 7246 & 178 & 0 & 2944 & 1.667 & 0 & 6.046 & 0 & 24.61 & 3.66 \\
\hline 92 & Smołdzino & & 260.29 & 7956 & 6737 & 131 & 1 & 159 & 1.647 & 0.015 & 82.390 & 0.629 & 5.57 & -4.12 \\
\hline 93 & Górno & \multirow{6}{*}{$\stackrel{x}{x}$} & 83.16 & 6786 & 977 & 201 & 1 & 3925 & 2.962 & 0.102 & 5.121 & 0.025 & 56.60 & -18.25 \\
\hline 94 & Masłów & & 85.55 & 4503 & 3260 & 432 & 0 & 8548 & 9.594 & 0 & 5.054 & 0 & 27.64 & 8.11 \\
\hline 95 & Łączna & & 61.65 & 2240 & 3521 & 374 & 2 & 6178 & 16.696 & 0.057 & 6.054 & 0.032 & 11.33 & 27.11 \\
\hline 96 & Bieliny & & 88.22 & 5840 & 2682 & 0 & 0 & 8809 & 0 & 0 & 0 & 0 & 41.20 & 0.40 \\
\hline 97 & Nowa Słupia & & 85.76 & 5768 & 2426 & 14 & 0 & 14 & 0.243 & 0 & 100 & 0 & 42.26 & -1.71 \\
\hline 98 & Bodzentyn & & 159.75 & 8185 & 7381 & 0 & 0 & 64 & 0 & 0 & 0 & 0 & 26.24 & 16.20 \\
\hline 99 & Poronin & \multirow{4}{*}{$\stackrel{x}{x}$} & 83.62 & 3331 & 4676 & 0 & 3 & 4954 & 0 & 0.064 & 0 & 0.061 & 14.83 & 25.92 \\
\hline 100 & $\begin{array}{l}\text { Bukowina } \\
\text { Tatrzańska }\end{array}$ & & 131.86 & 6372 & 5648 & 295 & 11 & 2225 & 4.630 & 0.195 & 13.258 & 0.494 & 23.32 & 12.83 \\
\hline 101 & Zakopane $^{*}$ & & 84.26 & 1927 & 4469 & 1120 & 5 & 3455 & 58.121 & 0.112 & 32.417 & 0.145 & -2.13 & 23.04 \\
\hline 102 & Kościelisko & & 136.68 & 3646 & 7263 & 0 & 0 & 4578 & 0 & 0 & 0 & 0 & 1.68 & 23.14 \\
\hline 103 & Dopiewo & \multirow{5}{*}{$\bar{x}$} & 108.02 & 7665 & 1742 & 0 & 0 & 1177 & 0 & 0 & 0 & 0 & 45.96 & -13.87 \\
\hline 104 & Puszczykowo* & & 16.39 & 211 & 804 & 43 & 6 & 574 & 20.379 & 0.746 & 7.491 & 1.045 & -12.13 & 19.05 \\
\hline 105 & Mosina & & 171.43 & 8203 & 6621 & 1005 & 33 & 4085 & 12.252 & 0.498 & 24.602 & 0.808 & 22.85 & 8.62 \\
\hline 106 & Komorniki & & 66.41 & 4389 & 1109 & 0 & 0 & 2921 & 0 & 0 & 0 & 0 & 41.09 & -13.30 \\
\hline 107 & Stęszew & & 175.02 & 12386 & 3213 & 0 & 0 & 1057 & 0 & 0 & 0 & 0 & 45.77 & -11.64 \\
\hline 108 & Krasnopol & \multirow{4}{*}{$\overline{\bar{x}}$} & 171.49 & 11240 & 3890 & 0 & 0 & 137 & 0 & 0 & 0 & 0 & 40.54 & -7.32 \\
\hline 109 & Nowinka & & 204.08 & 5691 & 12919 & \multicolumn{3}{|c|}{ incorrect data in BDL } & - & - & - & - & - & - \\
\hline 110 & Giby & & 323.2 & 5302 & 25089 & 0 & 0 & 0 & 0 & 0 & $0^{*}$ & $0^{*}$ & -8.60 & 47.63 \\
\hline 111 & Suwałki & & 264.61 & 13987 & 7886 & 1030 & 9 & 5544 & 7.364 & 0.114 & 18.579 & 0.162 & 27.86 & -0.20 \\
\hline 112 & Wolin & \multirow{3}{*}{$\overline{\bar{x}}$} & 327.46 & 15991 & 7496 & 23 & 0 & 1344 & 0.144 & 0 & 1.711 & 0 & 23.83 & -7.11 \\
\hline 113 & Świnoujście* & & 197.23 & 1753 & 4363 & 0 & 0 & 9729 & 0 & 0 & 0 & 0 & -16.11 & -7.88 \\
\hline 114 & Międzyzdroje & & 114.38 & 373 & 4986 & 1 & 12 & 238 & 0.268 & 0.241 & 0.420 & 5.042 & -21.74 & 13.59 \\
\hline
\end{tabular}

I - Ujście Warty NP, II - Babia Góra NP, III - Białowieża NP, IV - Biebrza NP, V - Bieszczady NP VI - Bory Tucholskie NP, VII - Drawa NP, VIII - Gorce NP, IX - Góry Stołowe NP, X - Kampinos NP, XI - Karkonosze NP, XII - Magura NP, XIII - Narew NP, XIV - Ojców NP, XV-Pieniny NP, XVI - Polesie NP, XVII - Roztocze NP, XVIII - Słowiński NP, XIX - Świętokrzyski NP, XX - Tatra NP, XXI - Wielkopolska NP, XXII - Wigry NP, XXIII - Wolin NP.

*_ cities which are part of a national park

restricted ${ }^{1}$. Among the analyzed rural municipalities, the largest area of changes of agricultural land for other purposes is planned in Stare Babice $(39 \%)$, which may result from urbanization around Warsaw.

In order to create a typology of the municipalities studied, they were divided into the following groups presented in Figure 1.

In terms of processes related to changes of agricultural land:

\footnotetext{
1 In accordance with Article 10a. of the Act on the protection of agricultural land and forestry land (consolidated text: Journal of Laws, 2017, Item 1161) restrictions on land changes for non-agricultural and non-forestry purposes shall not apply to agricultural land within the administrative boundaries of cities.
}

- the municipalities, where none of agricultural lands were changed for non-agricultural purposes - Group I -38 studied administrative units;

- the municipalities with absolute degree of changes of agricultural lands below 5\% Group II (53 units);

- the municipalities with absolute degree of changes of agricultural lands in range between $5 \%$ and $10 \%$ - Group III (8 units);

- the municipalities with absolute degree of changes of agricultural lands in range between $10 \%$ and $20 \%$ - Group IV (10 units);

- the municipalities with absolute degree of changes of agricultural land above $20 \%-$ Group V (4 units). 
- In terms of processes related to changes of forestry lands

- the municipalities, where none of forest lands were changed for non-forest purposes - Group 1 (71 administrative units);
- the municipalities with absolute degree of changes of forest lands below 2\% - Group 2 (40 units);

- the municipalities with absolute degree of changes of forest lands in range between $2 \%$ and $5 \%-$ Group 3 (1 unit);

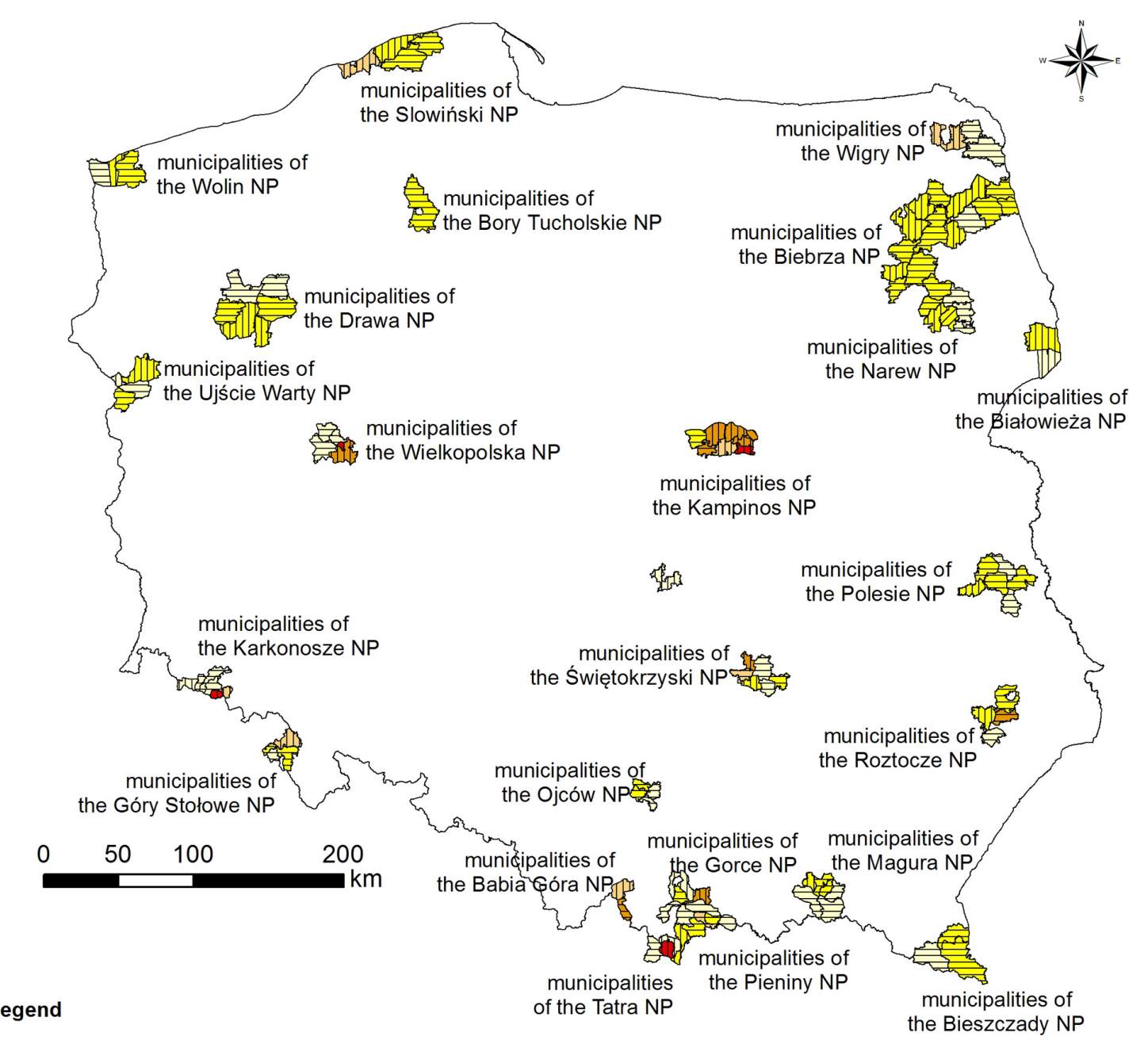

Groups of the municipalities by type of the processes related to changes of forest lands for non-forest purposes

Wchf

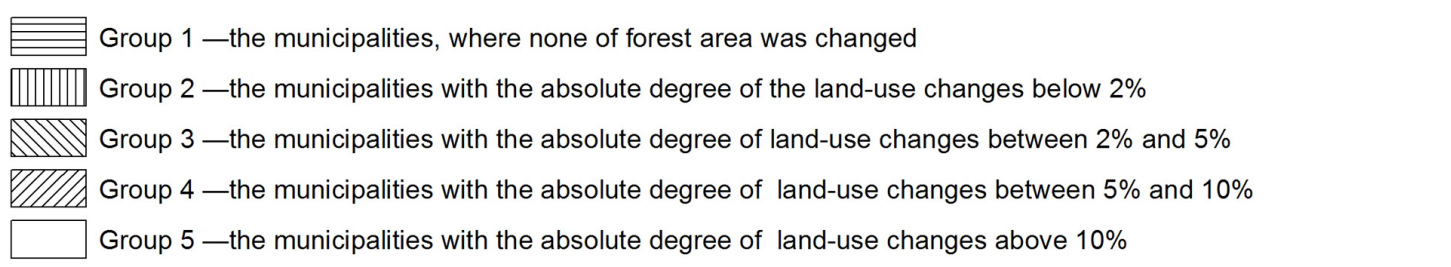

\footnotetext{
Groups of the municipalities by type of the processes related to changes of agricultural lands for non-agricultural purposes

Wcha

Group I -the municipalities, where none of agriculturalland was changed

Group II -the municipalities with the absolute degree of the land-use changes below $5 \%$

Group III - the municipalities with the absolute degree of land-use changes between $5 \%$ and $10 \%$

Group IV - the municipalities with the absolute degree of land-use changes between $10 \%$ and $20 \%$

Group V -the municipalities with the absolute degree of land-use changes above $20 \%$
}

Fig. 1. Groups of the municipalities according to the absolute degree of changes of agricultural and forest lands for non-agricultural or non-forestry purposes (own elaboration) 
- the municipalities with absolute degree of changes of forest lands in range between 5\% and 10\% -Group 4 (1 unit);

- the municipalities with absolute degree of changes of forest land above $20 \%$ - Group 5 (lack of units).

The analysis presents primarily the percentage share of agricultural and forestry landscape. It is considered to be important in terms of natural values and tourism development of the analyzed municipalities. In this case, the studied administrative units were assigned to one of four types, with respect to the minimal share of forestry and agricultural lands required for tourism (especially agritourism) development (Fig 2):

- type A - large surplus of agricultural or forest lands $-\mathrm{W}_{\mathrm{la}}\left(\mathrm{W}_{\mathrm{lf}}\right)>10 \%$;

- type B - small surplus of agricultural or forest lands $-0 \leq \mathrm{W}_{\mathrm{la}}\left(\mathrm{W}_{\mathrm{lf}}\right) \leq 10 \%$;

- type $\mathrm{C}$ - small deficit of agricultural or forest lands $-0<\mathrm{W}_{\mathrm{la}}\left(\mathrm{W}_{\mathrm{lf}}\right) \leq-10 \%$;

- type D - large deficit of agricultural or forest lands $-\mathrm{W}_{\mathrm{la}}\left(\mathrm{W}_{\mathrm{lf}}\right)<-10 \%$.

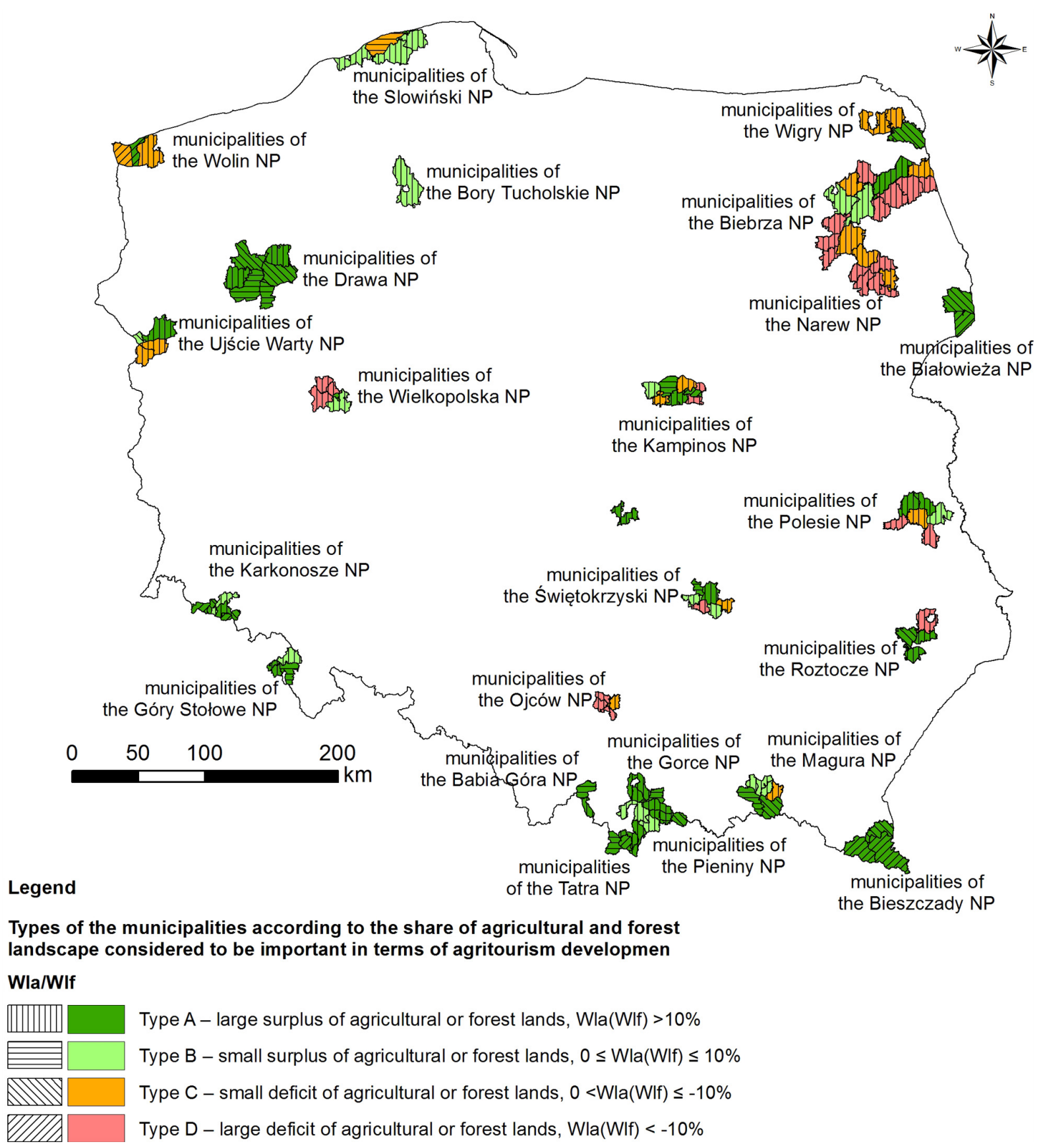

Fig. 2. Types of the municipalities according to the spatial coefficient of agricultural and forestry landscape (own elaboration) 
According to the types presented above, in relation to the share of forestry areas, 25 municipalities are assigned to the type $\mathrm{A}(22.1 \%), 18$ units $(16 \%)$ to the type B, 19 units $(16.8 \%)$ to the type $\mathrm{C}$ and 51 units to the type D (45.1\%).

\section{CONCLUSIONS}

Spatial diversification of the municipalities in terms of quantitative changes in the agricultural and forest land use is small but their location diversity in relation to the analyzed share of forest and agricultural landscape is high (Fig.1, Fig. 2). The spatial distribution can be observed in the case of regions and individual national parks.

In order to obtain a synthetic diversity picture of the municipalities in terms of their spatial policy related to agricultural and forest land a typology of the administrative units was developed. Tables 2 and 3 show the quantitative distribution of the designated types.

The most numerous set of the municipalities are the units that did not change the forest land for other purposes or did so to a minimal extent. Moreover, the share of forests in their area is a significant value in the context of tourism development. These are the municipalities assigned to the A.1 and A.2 categories.

A similar situation can be observed in the case of the municipalities classified into the B.1 and B.2 categories, in which forest areas are not the subject of changes introduced in spatial policy and moreover the share of forest land is slightly above the assumed minimum share of forests in the municipality.

On the basis of the obtained categorization, it can be observed that despite the lack of planning activities towards changes of forests for other purposes, in large number of the municipalities the share of forests is too low for the

Table 2. Quantitative distribution of the municipalities regarding of the forestry land changes for other purposes in the context of tourism development

\begin{tabular}{|c|c|c|c|c|c|}
\hline \multirow{2}{*}{ Group } & \multicolumn{4}{|c|}{ Type } & \multirow{2}{*}{$\Sigma$} \\
\hline & $A$ & $B$ & C & $D$ & \\
\hline 1 & 27 & 13 & 14 & 17 & 71 \\
\hline 2 & 24 & 6 & 4 & 6 & 40 \\
\hline 3 & 0 & 0 & 0 & 1 & 1 \\
\hline 4 & 0 & 0 & 0 & 1 & 1 \\
\hline 5 & 0 & 0 & 0 & 0 & 0 \\
\hline$\Sigma$ & 51 & 19 & 18 & 25 & 113 \\
\hline
\end{tabular}

requirements of agritourism development (C.1, C.2, D. 1, D.2 categories).

The extremely unfavorable situation in terms of planning decisions applied in local spatial development plans as well as in relation to the share of forests occurs in two municipalities: Łomianki (D.3) in the Kampinos National Park and Lapy (D.4) in the Narew National Park.

There is a considerably higher variation in the planning decision-making process in the case of the agricultural land. One can notice a significant advantage of the municipalities classified to the A.I and A.II categories in which the use of agricultural land for other purposes has not been changed or it has been done to a very small extent. In the municipalities classified to A.III, A.IV, A.V, B.III, B.IV categories, the share of agricultural landscape, despite planning decisions to reduce these area, is large enough to create favorable conditions for agritourism development. An extreme example is the municipality of Stare Babice, where there were very intensive changes of agricultural land for other purposes. However, the share of this kind of land-use is still high; therefore, the existing urban pressure has not changed either the agricultural character of the municipality or limited chances for agritourism development. In the case of the units classified to the CI, C.II, DI and D.II categories it should be noted that despite the protection of agricultural land in the planning process, the share of this kind of land-use in the land-use structure of the municipalities is below the requirements to be considered a positive factor determining the agritourism development.

A few but extremely negative cases are the following municipalities:

- Zakopane located in Tatra National Park, Karpacz in Karkonosze NP and Puszczykowo in Wielkopolska NP, in which there is a very dynamic process of changing agricultural land

Table 3. Quantitative distribution of the municipalities regarding changes of the agricultural land for other purposes in the context of tourism development

\begin{tabular}{|c|c|c|c|c|c|}
\hline \multirow{2}{*}{ Group } & \multicolumn{4}{|c|}{ Type } & \multirow{2}{*}{$\Sigma$} \\
\hline & A & $B$ & C & $D$ & \\
\hline 1 & 26 & 2 & 6 & 4 & 38 \\
\hline II & 42 & 4 & 5 & 2 & 53 \\
\hline III & 6 & 1 & 1 & 0 & 8 \\
\hline IV & 6 & 3 & 0 & 1 & 10 \\
\hline V & 1 & 0 & 0 & 3 & 4 \\
\hline$\Sigma$ & 81 & 10 & 12 & 10 & 113 \\
\hline
\end{tabular}


for other purposes together with a very small overall area of the agricultural landscape;

- Izabelin in Kampinos National Park with a significant decrease in agricultural land use for other functions.

In the first case, the existing trends are clearly associated with the municipal status of the administrative unit and lighter procedures concerning the changes of agricultural land for other purposes, regardless of the class of agricultural land. The second example shows the municipality with forest character affected by strong urban pressure. In this case, the forest protection takes place and at the same time all possible agricultural lands are undergoing urbanization.

\section{CONCLUSION}

1. The analyzed municipalities are a specific set of areas where natural conditions are one of the most important bases to be considered in the local spatial policy. The municipalities located within national parks are complex objects, which face complex socio-economic phenomena that in turn have the causal link on the sustainable development of these units. It should be emphasized that in most of the municipalities located within impact area of national parks, the provisions of the existing spatial development plans do not adversely affect the forest and agricultural landscapes resources. Some small area reduction may result from the implementation of public purpose investments, e.g. roads. The studied objects, where the adverse trends occur, are mostly located in the impact of large cities, such as Warsaw in the case of municipalities of the Kampinos $\mathrm{Na}$ tional Park and Poznań in relation to the municipalities of the Wielkopolska National Park.

2. In conclusion, it should be stated that most of the analyzed municipalities implement the principles of sustainable development, among others, by protecting agricultural and forest resources against intensive land-use changes for other purposes.

3. Paper published as a part of the project "IV Scientific and Technical Conference - Innovations in geodesy and cartography, real estate management and surface water protection", Janów Lubelski, Poland, 5-7 June 2019

\section{REFERENCES}

1. Borys T. 1999. Eco-development indicators. Wydawnictwo Ekonomia i Środowisko, Białystok.

2. Chmielewski T.J. 2001. A spatial planning system that harmonises nature and the economy, Wydawnictwo Politechniki Lubelskiej, Lublin.

3. Drzewiecki M. 1992. Wiejska przestrzeń rekreacyjna, Warszawa: Instytut Turystyki.

4. Drzewiecki M. 2005. Agroturystyka w Polsce-stan obecny i tendencje rozwojowe. In: B. Sawicki, J. Bergier (Eds.) Uwarunkowania rozwoju turystyki wiejskiej związanej z obszarami wiejskimi, Wydawnictwo PWSZ w Białej Podlaskiej, Biała Podlaska, 46-51.

5. Giordano K. 2006. Planning the commune's sustainable development in practice. Wydawnictwo KUL, Lublin.

6. Grochowska A. 2016. Spatial conflicts in spatial planning of metropolitan areas on the example of the Wrocław Metropolitan Area, Rozprawy Naukowe Instytutu Geografii i Rozwoju Regionalnego Uniwersytetu Wrocławskiego, Wrocław, pp. 240.

7. Heffner K., Klemens B. (Eds.) 2016. Rural Areas - Countryside Expanse and Population, Social Activity and Enterpreneurship, Studia, Vol. CLXVII, KPZK PAN, Warszawa, pp. 431.

8. Kołodziejczak A. 2017. Spatial Effects of the Desagrarization Process in the Rural Areas in Poland. Biuletyn KPZK PAN, Vol. 267, 75-83.

9. Kołodziejczak A., Kacprzak E. 2016. Funkcje rolnicze. In: Kaczmarek T., Mikuła Ł. (Eds.) Koncepcja kierunków rozwoju przestrzennego metropolii Poznań, Centrum Badań Metropolitalnych, Poznań, 75-83.

10. Kołodziejczak A., Kaczmarek L. (red.) 2018. Rozwój zintegrowanych obszarów wiejskich a ochrona zasobów przyrodniczych. Bogucki Wydawnictwo Naukowe, Poznań, pp. 161.

11. Kołodziejczak A., Głębocki B., Kacprzak E., Mackiewicz B., Szczepańska M. 2018. Social and Economic Costs of Spatial Disorder in Agriculture In: Kowalewski A., Markowski T., Śleszyński P. (Eds.) The costs of spatial disorder, Vol. II, Studia KPZK PAN, Vol. CLXXXII, 239-279.

12. Kostańska L. 2018. Institution of permanent and temporary exclusion of land from agricultural production in the process of investing in the agricultural production space. Człowiek i Środowisko, 40 (2), 65-78.

13. Krzysztofik M. 2016. Legal issues of registry of land and buildings in the context of the correct classification of agricultural land. Studia Iuridica Agraria XIV, 175-189.

14. Kwartnik-Pruc A., Parzych P., Bydłosz J. 2011. Problems of destination of agricultural and forest land for investment purposes in the country areas. Infrastructure and Ecology of Rural Areas, PAN Kraków, 4, 97-108 
15. Liszewski S. 2009. Tourist space of national parks in Poland. In: Domański B., Kurek W. (Eds.) Economy and space. Kraków, 187-201.

16. Markuszewska I., Marchewka P. 2016. Between economy and resource protection: management of agricultural land on selected examples in the Zbąszyń commune(Wielkopolska). Badania Fizjograficzne, VII, Seria A, Geografia fizyczna (A67), 133-143.

17. Pawłat-Zawrzykraj A, Podawca K. 2011. Sustainable social development of municipalities located in national park and their environmental protection, Ecological Questions 2011, Vol. 15, 81-90.

18. Podawca K., Karsznia K. 2017. The development of technical infrastructure between 2004-2014 as a factor of a sustainable development of the municipalities of Kampinoski National Park, Zeszyty Naukowe Inżynieria Środowiska, Seria Inżynieria Środowiska, Vol. 46, 85-95.

19. Podawca K., Mrozik K. 2019. Diversifying of the degree of implementation of planning and investment processes in the communes of the Warsaw Functional Urban Area, Scientific Review - Engineering and Environmental Sciences, 28(1), 105-117.

20. Podawca K. 2014. The analysis of sanitation services variation for communes under influence of national parks, Infrastructure and Ecology of Rural Areas, Vol. III/1, 985-999.

21. Podawca K. 2015. The analysis of socio-spatial availability of gas pipeline infrastructure for communes located in the impact zone of national parks. Economic and Regional Studies, 8(2), 67-79.

22. Podawca K., Pawłat-Zawrzykraj A. 2017a. Analysis of the availability of educational and technical infrastructure, as factor of sustainable development for Biebrza national park communes. Journal of Ecological Engineering, 18(1), 159-167.

23. Podawca K., Pawłat-Zawrzykraj A. 2017b. The analysis of agritourism accommodation indicators for areas located in vicinity of national parks. Annals of Warsaw University of Life Sciences SGGW, Land Reclamation, 49(4), 289-300.

24. Podawca K., Pawłat-Zawrzykraj A. 2018. Diversification of tourist attractiveness in municipalities located in impact area of national parks. Polish Journal of Environmental Studies, 27(5), 2213-2227.

25. Rosner A. (red.), 1999. Typology of rural problem areas, Polska Akademia Nauk-Instytut Rozwoju Wsi i Rolnictwa, Warszawa.

26. Sawicka Z., Fogel P. 2016. Functional Changes and Conversion of Agricultural Land in the Area with Fragmented Agrarian Structure, Wieś i Rolnictwo, 1(170), 165-184.

27. Suchoń A. 2016. The effect of the local spatial management plan on legal forms of agricultural real estates, Studia Iuridica Agraria XIV, 131-146.

28. Śleszyński P. (red.) 2013. Indicators of land use and spatial order in municipalities, Biuletyn KPZK PAN, 252, Warszawa.

29. Tanaś J. 2014. Non-agricultural Forms of Land Use in the Communes of Poznań Agglomeration. Journal of Management and Finance, 12(4), 437-455.

30. Wilkin J. (red.) 2018. The land is disappearing and subject to degradation the resources of agriculture and rural areas. Fundacja na rzecz Rozwoju Polskiego Rolnictwa (FDPA), Warszawa, pp. 78.

31. Zawilińska B., Mika M. 2013. National parks and local development in Poland: a municipal perspective. Human Geographies - Journal of Studies and Research in Human Geography, 7(1), 43-52.

32. Zielińska, A. (2013). Management on the Naturally Valuable Areas in Poland in the Context of Sustainable Development. Wrocław: Wydawnictwo Uniwersytetu Ekonomicznego we Wrocławiu, Seria: Monografie i Opracowania, Vol. 236. 